\title{
Fortgeschrittenes Schilddrüsenkarzinom: TKI-Therapie sorgfältig abwägen
}

\author{
Eine Therapieoption bei fortgeschrittenen Schilddrüsenkarzinomen sind \\ Tyrosinkinaseinhibitoren (TKI). Doch variieren Effekte und Nebenwirkungen \\ abhängig von Karzinomtyp und Substanz. In einer Metaanalyse werden die \\ Ergebnisse von Einzelstudien zusammengefasst.
}

D as fortgeschrittene Schilddrüsenkarzinom ist ein mögliches Einsatzgebiet für TKI. Studien mit verschiedenen Substanzen laufen oder wurden bereits abgeschlossen - ein direkter Vergleich einzelner Substanzen erfolgte bisher jedoch nicht. Hilfestellung für die Entscheidung, welche Substanz bei welchem Karzinomtyp die beste Option ist, liefern nun die Ergebnisse einer Metaanalyse. Forscher sammelten datenbankgestützt Studien, in denen TKI beim differenzierten (DTC) oder medullären Schilddrüsenkarzinom (MTC) mit einer Mindestnachbeobachtungszeit von 3 Monaten und primärem Endpunkt objektives Ansprechen untersucht worden waren. 22 Publikationen wurden identifiziert, die Daten extrahiert und u.a. in einer logistischen Regressionsanalyse zusammengefasst.

Das Ergebnis: Beim DTC erzielte Pazopanib mit $49 \%$ (95\%-Konfidenzintervall [95\%-KI] 33-64\%) die höchste gepoolte objektive Ansprechrate (ORR), gefolgt von Sorafenib mit $17 \%$ (95\%-KI 12-24\%). Gefitinib führte bei keinem Patienten mit DTC zu einem objektiven Ansprechen. Patienten mit MTC sprachen weder auf Gefitinib noch auf Imatinib objektiv an. Erfolgversprechender waren Sunitinib mit $43 \%$ (95\%-KI 14-77\%), Vandetanib mit $40 \%$ (95\%-KI $34-46 \%$ ) und Cabozantinib mit $27 \%$
(95\%-KI 22-32\%). Der klinische Nutzen ( „clinical benefit rate" $=$ Ansprechrate + Krankheitsstabilisierungen) als sekundärer Endpunkt errechnete sich für Patienten mit DTC unter Sorafenib mit $53 \%$ (95\%-KI 48-59\%) und für Patienten mit MTC unter Vandetanib mit $84 \%$ (95\%-KI 79-88\%) sowie unter Cabozantinib mit 55\% (49-61\%). Die Toxizität war bei allen TKI nicht unerheblich, häufige Nebenwirkungen waren Hand-FußSyndrome und gastrointestinale Effekte.

Fazit: Patienten mit fortgeschrittenen differenzierten oder medullären Schilddrüsenkarzinomen sprechen auf verschiedene TKI nicht oder mäßig an. Die Nebenwirkungen sind teilweise erheblich, sodass die Forscher den Einsatz dieser Substanzen nur für ausgewählte Patienten mit rasch progredienter Erkrankung empfehlen. Barbara Kreutzkamp

Klein Hesselink EN et al. Response and toxicity of small-molecule tyrosin kinase inhibitors in patients with thyroid carcinoma: A systematic review and meta-analysis. Eur J Endocrinol. 2015;172(5):R215-25.

\section{Junge Patienten mit Schilddrüsenkarzinom: Zervikale LK-Metastasen beeinflussen Prognose}

\author{
Die Prognose bei jüngeren Patienten mit papillären Schilddrüsenkarzinomen \\ galt bisher als gut, ungeachtet ihres lokoregionären Lymphknotenstatus. \\ Doch man sollte dies differenzierter beurteilen.
}

\footnotetext{
ᄃ in papilläres Schilddrüsenkarzinom ᄃ (PTC) bei Patienten unter 45 Jahren wird bisher als Niedrig-Risiko-Erkrankung eingestuft - auch wenn die Erkrankung lokoregionär fortgeschritten ist. In den aktuellen Stagingleitlinien des American Joint Committee on Cancer (AJCC) werden bei diesen Patienten die Krankheitsstadien lediglich entsprechend dem Vorliegen von Fernmetastasen unterschieden, was dem Stadium II entspricht, Patienten mit zervikalen Lymphknoten (LK)-Metastasen haben demnach eine Stadium-I-Erkrankung. Ein Grund für die bisherigen Empfehlungen ist wohl das Fehlen von aussagekräftigen Daten, u.a. zum Lymphknotenstatus, für die Prognose dieser Patientengruppe.
}

Das wurde nun mit einer Studie nachgeholt, in der Krankenakten von 1998 bis 2006 aus der National Cancer Data Base (NCDB) und dem Programm „Surveillance, Epidemiology, and End Results" (SEER) extrahiert wurden. Identifiziert wurden Patienten unter 45 Jahren, die wegen eines PTC im Stadium I operiert worden waren. Die NCDB steuerte 47.902 Patienten bei (11.740 mit und 36.162 ohne LK-Metastasen), die SEERDatenbank 21.855 Patienten (5.188 mit und 16.667 ohne positiven Nodalstatus). Nach Adjustierung zeigte sich bei Patienten mit zervikalen LK-Metastasen ein geringeres Gesamtüberleben als bei Patienten ohne lokoregionären LK-Befall (NCDB: Hazard Ratio [HR] 1,32;
95\%-Konfidenzintervall [95\%-KI] 1,041,$67 ; \mathrm{p}=0,021$; SEER: HR 1,29; $95 \%$-KI $1,08-1,56 ; p=0,006)$. Eine zunehmende Zahl metastasierter LK war mit einem sinkenden Gesamtüberleben assoziiert (HR 1,12; 95\%-KI 1,01-1,25; p = 0,03), erst bei $\geq 6$ positiven LK erhöhte sich das Risiko nicht mehr weiter (HR 0,99; $95 \%$-KI 0,99-1,05; $\mathrm{p}=0,75)$.

Fazit: Anders als bisher sollten auch Patienten unter 45 Jahren mit einem papillären Stadium-I-Schilddrüsenkarzinom entsprechend der Anzahl zervikal metastasierter Lymphknoten prognostisch stratifiziert werden. Bei lokoregionärem LK-Befall nimmt das Gesamtüberleben proportional zur Anzahl der positiven Lk ab, bei 6 LK wird ein Plateau erreicht. Diese Erkenntnisse sollten nun in neue Leitlinien eingearbeitet werden.

Barbara Kreutzkamp

Adam MA et al. Presence and number of lymph node metastases are associated with compromised survival for patients younger than age 45 years with papillary thyroid cancer J Clin Oncol. 2015;33(21):2370-5. 